\title{
DISENTANGLING CAUSAL PLURALISM
}

\author{
LEEN DE VREESE \\ Centre for Logic and Philosophy of Science \\ Ghent University, Belgium \\ E-mail: Leen.DeVreese@UGent.ac.be
}

\begin{abstract}
Causal pluralism is increasingly gaining interest as a promising alternative for monistic approaches toward causation. However, although the debate is scarcely out of the egg, the term 'causal pluralism' already covers diverse meanings. This creates confusion, and to remedy that confusion, it is necessary to discern different kinds of pluralistic approaches to causation and different possible positions within them. In this paper, I argue for a general distinction between conceptual causal pluralism, metaphysical causal pluralism and epistemological-methodological causal pluralism. I mainly focus on metaphysical approaches to causation and discern herein four possible positions: metaphysical causal constructivism, metaphysical causal monism, weak metaphysical causal pluralism, and strong metaphysical causal pluralism. Each of these positions are further related to their most obvious conceptual counterpart, specifically conceptual causal monism or conceptual causal pluralism.
\end{abstract}

\section{Introduction}

Traditionally, philosophers concerned with causation have been reasoning from monistic presuppositions. They have supposed that causation is a univocal concept referring to a single kind of relation in the world. Recently, alternative approaches have emerged within the debate on causal pluralism. Causal pluralism indeed forms a promising alternative to causal monism, and the interest for this alternative view is steadfastly growing. However, although the literature on 'causal pluralism' is altogether still limited, the term already covers diverse meanings within this current literature. This creates confusion, which thwarts the fruitful development of this increasingly significant approach to causation. To remedy that confusion, it is necessary to discern different kinds of pluralistic approaches to causation and different possible positions within them. Therefore, I will try to make a good start in structuring and clarifying the debate, by introducing some distinctions in this paper.

Generally, I discern three kinds of causal pluralism: conceptual causal 
pluralism, metaphysical causal pluralism, and epistemological-methodological causal pluralism. Each of these oppose their monistic counterparts. The literature in defense of causal pluralism focuses particularly on the first kind of causal pluralism: conceptual causal pluralism. Authors subscribing to conceptual causal pluralism maintain that our everyday notion of 'causation' cannot be described univocally, while authors subscribing to conceptual causal monism maintain that it can. I will briefly revise the arguments in defense of causal pluralism in section 2 .

The main aim of this paper is to clear up part of the confusion in the causal pluralism debate by discerning different possible positions with respect to metaphysical causal pluralism. This will be done by means of three central metaphysical questions concerning causation. Firstly, is causation a realistic notion, or is it a mental construct? Secondly, does causation only occur as a real relation at the fundamental level of reality, or does it also occur as a real relation between objects at higher levels of reality? And lastly, does causation consist in a single empirical relation, or does it consist in diverse empirical relations deserving the label 'causal'? The possible answers to these questions lead to different metaphysical positions, which will be expounded and illustrated in section 3. Arguing for one or another metaphysical position is nonetheless not noncommittal. It carries implications for one's conceptual approach to causation. In section 4, I will present the most obvious complementary conceptual approaches to the different metaphysical positions. Metaphysical causal pluralism will be discerned from epistemological-methodological causal pluralism in section 5, where I will briefly comment on the relations between the former and the latter. Section 6 will contain my final conclusions.

\section{Conceptual causal pluralism}

Concerning our everyday notion of causation, only one central question guides the choice for or against causal pluralism: is our everyday notion of causation monistic or pluralistic? Since I am here concerned with causal pluralism, I will focus on the arguments in defense of conceptual causal pluralism. The following three arguments take a central place in the current literature defending conceptual causal pluralism:

1. All available monistic approaches to causation (e.g., manipulation approaches, probabilistic approaches, causal mechanism approaches, counterfactual approaches, etc.) have counterexamples and restrictions. Some of these approaches are even clearly inapplicable to cer- 
tain domains of knowledge. (See e.g., Ref. 1.)

2. In some cases, even our everyday intuitions on whether a certain factor is really the cause of an event are ambiguous. If we would have a clearly outlined, univocal notion of 'causation' at our disposal, this would not be the case. (See Ref. 2.)

3. In everyday reasoning and decision-making, we do not need to know what the 'real' causes are, for instance whether a probabilistic approach or rather a counterfactual approach points to the 'real' causal relations in the world. In practice, it mostly suffices to know that factor $\mathrm{C}$ is for example counterfactually dependent on factor $\mathrm{E}$, or that there is a causal mechanism connecting $\mathrm{C}$ and $\mathrm{E}$, etc. One does not need to know then whether these properties also define 'real' causation. (see Ref. 2.)

It is the first kind of argument, for example, which has led Ned Hall to conceptual causal dualism. ${ }^{1}$ Specifically, well-known counterexamples to the counterfactual analyses of causation have led Hall to a distinction between causation as dependence and causation as production:

"Causation, understood as a relation between events, comes in at least two basic and fundamentally different varieties. One of these, which I call 'dependence,' is simply that: counterfactual dependence between wholly distinct events. In this sense, event $c$ is a cause of (distinct) event $e$ just in case $e$ depends on $c$; that is, just in case, had $c$ not occurred, $e$ would not have occurred. The second variety is rather more difficult to characterize, but we evoke it when we say of an event $c$ that it helps to generate or bring about or produce another event $e$, and for that reason I call it 'production'."

(Ref. 1, p. 225)

What forms the basis for this distinction made by Hall? His analysis starts with those causal relations that form a counterexample for the counterfactual theory of causation, namely cases of overdetermination. Counterfactual dependence cannot form an argument for causation in these cases, since several factors ensuring the effect are simultaneously present:

"Suzy and Billy, expert rock-throwers, are engaged in a competition to see who can shatter a target bottle first. They both pick up rocks and throw them at the bottle, but Suzy throws her a split second before Billy. Consequently Suzy's rock gets there first, shattering the bottle. Since both throws are perfectly accurate, Billy's 
would have shattered the bottle if Suzy's had not occurred, so the shattering is overdetermined." (Ref. 1, p. 235)

In this case, Suzy's throw is a cause of the shattering, but Billy's throw is not. The shattering nonetheless does not depend on Suzy's throw. Since if Suzy's throw would not have caused the bottle to shatter, it had shattered anyway thanks to Billy's throw. Hall argues that one should add three theses on causality to solve this problem: the thesis of transitivity, the thesis of locality, and the thesis of intrinsicness. The first states that causation is a transitive relation, the second that 'causes are connected to their effects via spatiotemporally continuous sequences of causal intermediates', and the latter that 'the causal structure of a process is determined by its intrinsic, non-causal character' (Ref. 1, p. 225). These three theses make up for causation as production. The example above is indeed a case of 'pure production', in which dependence is of no concern.

However, in order to be able to deal with cases of double prevention and omission, one should throw the three additional theses overboard. In these cases, counterfactual dependence is the only thesis that matters. Hall gives the following example of 'pure dependence':

"Suzy and Billy have grown up, just in time to get involved in World War III. Suzy is piloting a bomber on a mission to blow up an enemy target, and Billy is piloting a fighter as her lone escort. Along comes an enemy fighter plane, piloted by Enemy. Sharpeyed Billy spots Enemy, zooms in, pulls the trigger, and Enemy's plane goes down in flames. Suzy's mission is undisturbed, and the bombing takes place as planned. If Billy hadn't pulled the trigger, Enemy would have eluded him and shot down Suzy, and the bombing would not have happened." (Ref. 1, p. 241)

In this example, the effect counterfactually depends on the cause, but there is no mechanism linking cause and effect. Billy's pulling the trigger did not produce the bombing, but it was nonetheless necessary for Suzy to be able to execute the bomb attack. Hence, the occurrence of the bombing was dependent on Billy's pulling the trigger.

The conclusion Hall draws from this analysis is that counterfactual dependence captures only one kind of causal relation, and that another kind of causal relation exists which needs only the theses of transitivity, locality and intrinsicness but not the thesis of counterfactual dependence. The former are dependence relations, while the latter are production relations. 
However, typical causes are cases of dependent production rather than pure production or pure dependence. So although production and dependence are conceptually distinct, in the actual world their extensions overlap in most cases. This also clarifies why both kinds of causal relations are easily jumbled up and a single kind of causal relation is generally supposed.

\section{Three metaphysical questions}

Let me turn to the central question of this paper: what about metaphysical causal pluralism? In other words, do metaphysical reasons exist to consider causal pluralism, or is pluralism with respect to causation only a conceptual matter? I discern three central metaphysical questions which should be answered, and which lead quasi-automatically to certain positions with respect to the causal pluralism debate. A first question is whether causation is a real relation, or rather a mental construction. The answer to this question makes it possible to discern realistic from constructivist views on causation. Realists should answer a second question, namely whether 'causation' refers to a single kind of empirical relation, or rather refers to different kinds of empirical relations all labeled 'causal'. I will call the latter position 'strong metaphysical causal pluralism'. If one maintains, as most philosophers do, that 'causation' refers to only one kind of empirical relation in the world, the answer to a last question can determine whether one is a metaphysical causal monist on the one hand, or rather a weak metaphysical causal pluralist on the other hand. Namely, the question whether 'causation' refers to a relation which only exists between elements at the elementary level of reality on which all other causal relations then supervene, or whether causation is a real relation between all kinds of objects at all levels of reality. In the following subsections, I will have a closer look at the possible answers to these three questions and the resulting positions toward metaphysical pluralism.

\subsection{Causation as a realistic notion versus a mental construct}

A first metaphysical question to be posed is whether causation is not just a mental construct created by mankind. Is there after all some real kind of relation in the world to which our concept of 'causation' refers? Hume's famous view on causation calls this into question, ${ }^{3,4}$ by asserting that causation is nothing more than constant conjunction in combination with temporal priority of the cause and spatiotemporal contiguity between cause 
and effect. It is the memory of past co-occurrences of the cause and effect that lead in new instances of the cause to the supposition that the effect will follow, and hence to the supposition of a constant conjunction between the cause and effect. However, men further suppose that the connection between cause and effect is necessary, but this necessity cannot be inferred from our perceptions. This convinced Hume that, from a philosophical point of view, causation has to be solely understood in terms of temporal priority, spatiotemporal contiguity and constant conjunction, and not in terms of a necessary connection. The idea of the necessity of a causal relation is a further construction of the human mind upon the more objective description of causation in terms of constant conjunction, temporal priority and spatiotemporal contiguity, but this latter description is closest to our perceptions and hence the one that enables consensus on what causation is.

A similar view has more recently been proposed by Jon Williamson..$^{5,6}$ In his view labelled epistemic causality, causation is also believed to be a mental construct. Williamson starts from the assertion that it is just handy for people to think in terms of cause and effect, and that this is also the reason why they do so, and not that there is something physical corresponding to the term 'cause'. Williamson is nonetheless convinced that there is an objective reference point for the justification of our causal claims, namely the fullest knowledge of the world. The different views on causation as outlined in different causal theories, form what he calls different causal indicators. However, this variety of causal indicators does not imply a variety of concepts of causation, according to Williamson. Our causal beliefs are based on these several indicators, but all knowledge from all these indicators of causality would lead together to the fullest knowledge of the world. And it is precisely this fullest knowledge of the world which forms the objective reference point of what causation is, and which would deliver a monistic epistemic concept of causation. To sum up, Williamson maintains that causality is a feature of our epistemic representation of the world, and not a real part of the world itself. According to his view, causation further does not consist of a variety of concepts, but is rather one eclectic notion. Hence, just like Hume, Williamson defends the metaphysical view that causal relations are no real part of the world, but relations constructed on this reality by men. I will call this kind of position metaphysical causal constructivism. The real challenge for philosophers defending a constructivist metaphysical approach, is to clarify why it is nonetheless successful and/ or necessary to think in terms of cause and effect.

In the case one defends the opposite position known as causal realism - 
namely that there ís something physical in the world to which our notion of 'cause' refers - further metaphysical questions need to be answered. These questions and their possible answers will be expounded in the next two sections. To conclude this section, I first specify what causal realism is. In its strongest interpretation, causal realism can be described as follows:

"Realism about causation requires two things. First, according to the realist, causation is objective, meaning that it is something that occurs in an 'external reality' as opposed to something that is merely subjective, a feature of our thoughts or perceptions alone (that is, merely an idea or a concept). The distinction between objective and subjective causation thus concerns the issue of whether or not causation is mind-independent. Second, according to the realist, causation involves some sort of necessity with respect to the connection between causes and effects. ...

For now, let it suffice to say that by invoking necessity, realists of different stripes maintain that there is more to causation than mere constant or probabilistic conjunctions of events. Merely subjective accounts of causation hold that if there is such a thing as causal necessity, it is an idea or a concept only. Objective accounts hold that there is such a thing, and that it is a feature of the world quite apart from our ideas or concepts." (Ref. 7, p. 8)

I think this definition captures clearly what causal realism involves. I have nonetheless presented the theories of Hume and Williamson above which both maintain that causation is a mental construction that can nonetheless be conceived as objective. Consequently, the definition would still be clearer when the terms 'objective' and 'subjective' were replaced with respectively 'not-mental' and 'mental'. Further, the reverse claim can be found as well: Huw Price defends in Refs. 8 and 9 a view on causation which holds that causality is not a mental notion, but meanwhile neither fully objective. Price's approach results in a rather weak version of causal realism. Regardless of whether one defends causal realism in a strong or in a rather weak sense, if it is accepted that something in the world exists to which our notion of cause refers, two further metaphysical questions obtrude themselves. 


\subsection{Causation as a single versus a plural empirical relation}

A second metaphysical question is whether our notion of 'cause' refers to a single kind of relation in the world or whether several kinds of empirical relations have to be discerned, although they are all labeled 'causal'. This last option has been advanced by Ned Hall in Ref. 1. Hall interprets his distinction between production and dependence in the first place as a conceptual distinction, as it was presented in section 2. He nonetheless explicitly mentions the possibility to interpret his view as one making a metaphysical distinction:

"A more subtle objection is the following: What I have really shown is not that there are two concepts of causation, but rather that there are two kinds of causation, two different ways in which one event can be a cause of another. That may well be right; certainly, I was happy to begin this paper by announcing that event-causation comes in two 'varieties.' I do not know how to judge the matter, because I am not sufficiently clear on what underlies this distinction between concepts and kinds. ...

I am quite content to agree that I have (merely) shown that there are two kinds of causation - as long as those who insist on this rendering of my thesis agree that the two kinds answer to very different criteria and consequently require very different analyses."

(Ref. 1, p. 255-256)

Hall even advances the possibility that further research will demonstrate that even more empirical kinds of causal relations should be discerned. As far as I know, Hall is currently the only philosopher explicitly defending a dualistic position toward causation that can be metaphysically interpreted. It is clearly not at all easy to substantiate this kind of position, which I like to label strong metaphysical causal pluralism. Consequently, the opposite answer, namely that causation is a single kind of empirical relation, remains the most obvious view. John L. Mackie as well as Phil Dowe, for example, described causation as a single kind of empirical relation. However, a last metaphysical question divides their views.

\subsection{Causation as a relation between elements at the fundamental versus at all levels of reality}

The last metaphysical question is whether causation is a relation only existing at the fundamental level of reality on which other 'causal relations' 
then supervene, or whether causation has to be interpreted as a real relation between all kinds of objects at all levels of reality. Phil Dowe follows the former route in the view on causation presented in Physical Causation. ${ }^{10}$ In this book, Dowe defends an approach to causation which is based on Salmon's process theory:

"The approach to be taken is to modify Salmon's theory by introducing the concept of a conserved quantity. The central idea is that it is the possession of a conserved quantity, rather than the ability to transmit a mark, that makes a process a causal process." (Ref. 10, p. 89)

This leads to the following central claims of Dowe's own process theory:

CQ1. A causal interaction is an intersection of world lines which involves exchange of a conserved quantity.

CQ2. A causal process is a world line of an object which possesses a conserved quantity.

A 'conserved quantity' is any quantity which is universally conserved, and current scientific theory is our best guide as to what these are. Thus, we have good reason to believe that massenergy, linear momentum, and charge are conserved quantities.

(Ref. 11, p. 323)

Dowe's theory of causation in terms of 'conserved quantities' raises several questions. Most importantly, although Dowe presents his own, rather physical approach as superior to its opponents, it is not at all clear how to apply it to the knowledge from other scientific domains. Some information which he only obliquely hints to is crucial to understand Dowe's point of view. It turns out that he underpins his approach to causation with a reductionistic metaphysical position:

"... One answer is that the generality of 'conserved quantity' might allow this to be used as a testable conjecture in various fields of science. But it is unlikely that it would stand the test: conservation laws seem to be confined to the physical sciences. A more desirable option is to take a middle road and adopt a supervenience account such as that of $\mathrm{Kim}^{12}$ where causes supervene on conserved quantities. ${ }^{13 "}$ (Ref. 14, p. 214-215) 
Peter Menzies summarizes this view of Jaegwon Kim, on which Dowe founds his own approach of causation, as follows:

"There is a supervenient causal relation, Kim tells us, between the $a$ 's having $\mathrm{F}$ and b's having $\mathrm{G}$ just in case there are two events $a$ 's having $F^{*}$ and $b$ 's having $G^{*}$ such that $a$ 's having $\mathrm{F}$ supervenes on $a$ 's having $F^{*}, b$ 's having $\mathrm{G}$ supervenes on $b$ 's having $G^{*}$, and $a$ 's having $F^{*}$ causes $b$ 's having $G^{*}$. It is Kim's view that all macrolevel causal relations are supervenient causal relations of this kind, where the causal relations at the base level relate microlevel events. . . He sees the rationale for his view that all macrolevel causal relations are supervenient causal relations as lying in what he calls the thesis of microdeterminism, according to which the world is the way it is because the microworld is the way it is. He sees this thesis as urging us to look on the causal order of the macroworld as emerging out of the causal order of the microworld." (Ref. 13, p. 554)

This metaphysical premise, according to which causation in the world has to be reduced to the domain of physics, clarifies the whole argumentation behind Dowe's position in Physical Causation. I label this position which maintains that our notion of 'causation' refers to one kind of empirical relation at a single, basic level of reality metaphysical causal monism. The challenge for metaphysical causal monists, such as Dowe, lies in the justification of their metaphysical point of view. An important argument in defense of this point of view is that we should accept it if we take our physicalistic world view seriously. ${ }^{12}$ This argument is opposed by philosophers substantiating the claim that physicalism accommodates an ontological preconception with respect to physics which cannot be united with scientific knowledge and scientific practice (e.g., Stephen Webster, ${ }^{15}$ Nancy Cartwright, ${ }^{16}$ Michael Silberstein $\left.{ }^{17}\right)$ :

"The commonest justification of this position is simply to point out that higher level phenomena often cannot be predicted from a knowledge of the component part that constitute the lower level. Take water for example: its behaviour cannot be predicted simply from a knowledge of hydrogen and oxgyen [sic.]. For the antireductionist, this inability is not simply a result of ignorance. It is a matter of principle that every level has its own characteristic patterns and processes, particular to that level, and not replaceable by the patterns and processes of a lower level." (Ref. 15, p. 54) 
The latter conviction does not need to imply strong metaphysical causal pluralism, but can be accommodated with a position in between strong metaphysical causal pluralism and metaphysical causal monism, which I label weak metaphysical causal pluralism. This position entails that causation is a single kind of relation, which nonetheless occurs at all levels of reality as a real, and hence not-reducible, relation. John L. Mackie's approach seems to belong to this category. Mackie defines causation, after John Stuart Mill, as a complex regularity:

"In $\mathrm{F}$, all $(A B \bar{C}$ or $D G \bar{H}$ or $J K \bar{L})$ are followed by $\mathrm{P}$, and in $\mathrm{F}$, all $\mathrm{P}$ are preceded by $(A B \bar{C}$ or $D G \bar{H}$ or $J K \bar{L})$.... That is, some disjunction of conjunctions of factors, some of which may be negative, is both necessary and sufficient for the effect in the field in question." (Ref. 18, p. 63)

In this definition, negative causes are presented formally as $\bar{X}$. Further, $F$ represents the causal field, which forms the background of the causal event, but is no part of the cause itself according to Mackie. He maintains that a theory trying to describe what causality is in the world, has to be concerned with these whole complex regularities. Although Mackie is further not very explicit about his metaphysical position, it becomes clear from his account that he would defend that causation consists of a single kind of empirical relation, in line with his conceptual approach. Unlike Dowe, he seems nevertheless convinced that this relation is present as a real relation in all domains of reality, what makes Mackie a weak metaphysical causal pluralist:

"I insist that our concept is in several ways a bit indeterminate: 'cause' can mean slightly different things on different occasions, and about some problematic cases, for example of over-determination, we may be unsure what to say. But it is still a fairly unitary concept: we do not have one concept for physical causation and another for human actions and interaction (as someone might be forced to say who took our concept of physical causation to be that of regular succession); we can and do assert similar counterfactual necessity (and at times sufficiency) about fields of all different sorts." (Ref. 18, p. xi) 


\section{The relations between metaphysical and conceptual positions}

In the previous section, I discerned four possible metaphysical positions toward causation: metaphysical causal constructivism, strong metaphysical causal pluralism, weak metaphysical causal pluralism and metaphysical causal monism. Arguing for one or another of these positions is not noncommittal. It carries implications for one's conceptual approach to causation. Let me run through the various metaphysical positions again to point out what the most obvious complementary conceptual approach would be.

In John Williamson's case, it is the specific form of his theory in terms of epistemic causality which leads to what I interpret as a kind of conceptual causal pluralism, namely the conviction that we refer to a unitary epistemic concept by way of divergent causal indicators. Jon Williamson himself states that his view forms an improved monistic approach to causation, which has the advantage of being able to deal with the epistemic usefulness of a variety of 'causal indicators' (causal mechanisms, counterfactuals, correlations, etc.). I think this interpretation of his own view on causation should be refined by introducing a distinction between conceptual causal monism/pluralism and epistemological causal monism/pluralism ${ }^{\mathrm{a}}$. The term 'conceptual' is used as before to refer to our everyday concept(s) of causation, which guide(s) the way(s) we come to our everyday causal judgements. I further use the term 'epistemological-methodological' to refer to the meaning of causation from a knowledge point of view. I would prefer then to interpret Williamson's approach as a combination of conceptual causal pluralism and epistemological causal monism: in daily practice, we make use of a variety of causal indicators, but once we would possess the fullest knowledge of the world, one concept of causation would reveal itself from it. Consequently, causal theories explicate different everyday interpretations of the notion 'cause', that nonetheless indicate together a single - be it eclectic - epistemic concept. Another filling-in of a metaphysical causal constructivist theory of causation can just as well lead to conceptual causal monism. Hume maintained for example that only one concept of cause (in terms of spatiotemporal contiguity, temporal priority and constant conjunction) is good enough to serve as our concept of cause ${ }^{3}$ since the other ways in which we are inclined to capture our notion of cause face epistemological problems. This revisionistic stance of Hume

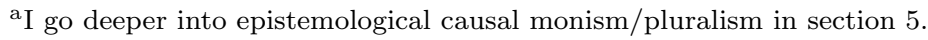


gets him to defending a monistic conceptual approach to causation on the basis of his constructivist metaphysical position. Hence, the choice for or against conceptual causal pluralism is in the case of metaphysical causal constructivism entirely dependent on the specific premises of the causal theory defended.

A thoroughly substantiated strong metaphysical pluralism, on the other hand, automatically forms a strong argument for the necessity of conceptual causal pluralism. Discerning production and dependence as two different kinds of causal relations in the world, as Ned Hall proposes, ${ }^{1}$ implies the need to have at least also this distinction in our conceptual apparatus in order to make appropriate causal judgements.

Even so does a thoroughly substantiated metaphysical monism automatically form an argument in defense of conceptual causal monism. Hence, Phil Dowe's conviction that causation only consists of a unique empirical relation at the fundamental organizational level of the world necessitates him to be revisionistic. ${ }^{14}$ His metaphysical conviction grounds the way he rejects all alternative approaches to causation in favor of his own approach in terms of causal processes and conserved quantities. In Ref. 19, Dowe nonetheless agrees that omissions and preventers can in practice not always easily be distinguished from genuine causes, although they are not physically connected to their effects and consequently are no genuine causes according to his view. Dowe labels them 'quasi-causes', and states that it is even useful practically to treat quasi-causation as genuine causation. Furthermore, causation and quasi-causation seem to play very similar practical roles. According to Dowe, the unity of both lies in the fact that quasi-causation is, in essence, possible causation. Distinguishing them is nonetheless important theoretically. Since 'causation' appears in the definition of what 'quasi-causation' is, they cannot be treated as being the same. Hence, a description of genuine causation should throw cases of quasi-causation overboard. It follows that we should revise our notion of cause, such that quasi-causes are not included in our description.

The most natural complement of weak metaphysical causal pluralism is also conceptual causal monism. In the quotation at the end of the former section, Mackie, for instance, clearly argues for a single, unitary concept applicable throughout all possible domains of application.

However, things are not that straightforward, and some further comments are needed. The first comment concerns pragmatical considerations. Mackie creates some place for play which has to accommodate his view to pragmatical differences in causal judgements. According to his theory, in 
which a cause is supposed to be the factor which is necessary in the circumstances for the effect to occur, differences in everyday causal judgements can be attributed to the way we select 'the' cause from the factors of the disjunction of conditions:

"The supposed distinction between conditions and causes can be adequately accounted for in these two ways: an alleged condition which is not called a cause, although if in the circumstances it had not occurred the result would not, either is part of the field presupposed in the view taken by the speaker of the result (and so is not a cause in relation to this field) or is a cause, but mention of this fact happens to be irrelevant, or less relevant than mention of some other cause of the same result, to some current purpose." (Ref. 18, p. 36)

Pragmatical factors do not affect Mackie's basic concept of cause, although they can affect the causal judgements resulting thereof. Apart from Mackie's approach, pragmatical considerations can also be introduced to justify the defense of a monistic conceptual approach where a pluralistic approach seems more obvious and the reverse. Some metaphysical approaches seem to leave more room for this kind of considerations than others. Weak conceptual causal pluralism as well as metaphysical causal monism seem to be approaches that can easily be combined with pragmatically based conceptual causal pluralism. For example with regard to Dowe's theory, one could argue that we will need a variety of concepts of causation for everyday causal reasoning as long as we do not possess enough knowledge to determine how specific causal relations in the macroworld depend on their underlying physical constitution. On the other hand, it seems much less obvious to argue for a combination of strong metaphysical causal pluralism with pragmatically based conceptual causal monism. However, the central conclusion is that it is utterly important to provide thorough pragmatical arguments to underpin atypical combinations.

Further, one position which is possible in principle has nonetheless not been discussed. It forms no obvious point of view and is nowhere defended in the literature, as far as I know. It concerns a metaphysical position which defends that all causal relations have to be reduced to the elementary level but that diverse empirical kinds of causal relations nonetheless exist at that basic level. The most natural conceptual complement of such a position would clearly be conceptual causal pluralism. 


\section{Epistemological-methodological causal pluralism}

To conclude, I briefly indicate the importance of discerning conceptual and metaphysical causal pluralism from a third kind of approach to causal pluralism, namely what I label epistemological- methodological causal pluralism. The latter refers to the importance of a pluralistic view on causation for our scientific knowledge in general on the one hand, and for assembling causal knowledge in specific domains of science on the other hand. It will be clear that an epistemological-methodological approach to causation is not disconnected from conceptual and metaphysical approaches to causation. It is nonetheless important to value this line of approach as different from the others. Especially in the case of conceptual and/or metaphysical pluralism, certain questions become utterly important for the sake of our knowledge. What is the best way to acquire causal knowledge? Do the useful concepts of causation differ from domain to domain? Do we need a variety of causal concepts to achieve sufficient causal knowledge within one domain? What are the useful concepts of causation for the different scientific domains? If causation is a single empirical relation, why do we possibly need a variety of causal concepts to gather scientific knowledge? Do scientists make use of a variety of concepts in practice? The answers to these questions will be affected by the conceptual and especially metaphysical position taken, but can on their turn affect the way causal knowledge is gathered and subsequently what causal knowledge is reached. This demonstrates again that arguing for certain conceptual and/or metaphysical positions, is not at all a noncommittal activity.

\section{Conclusion}

'Causal pluralism' is a very broad notion, covering entirely divergent approaches to causation. The conviction that causation is no single, univocal thing can lead to a wide area of alternative 'pluralistic' approaches to causation. Consequently, I argued that the confusion in the current debate on causal pluralism should be avoided by refining our view on causal pluralism. In the first place, one should make a clear distinction between three ways to approach the debate: from a conceptual point of view, from a metaphysical point of view or from an epistemological-methodological point of view. I focused in this paper on metaphysical approaches to causation and discerned four possible metaphysical positions: metaphysical causal constructivism, metaphysical causal monism, weak metaphysical causal pluralism and strong metaphysical causal pluralism. Each of these positions can be 
related to the most obvious conceptual counterpart, i.e., conceptual causal monism or conceptual causal pluralism. It is utterly important precisely to determine one's position in the debate and to become aware of mutual connections between certain positions in order to improve the discussion. I hope to have offered a general framework that can help in creating more clarity. The further development of appropriate and fruitful pluralistic approaches should benefit from a refined view on what 'causal pluralism' can consist in. On the other hand, it is evident that the framework itself may also need further development in the course of the discussion, when new lines of argumentation are developed.

\section{Acknowledgments}

I am greatly indebted to Erik Weber and the referee for their insightful comments on earlier versions of this paper. The research for this paper was supported by the Research Fund of the Ghent University through research project BOF2001/GOA/008 and by the Fund for Scientific ResearchFlanders through research project G.0651.07.

\section{Bibliography}

1. N. Hall, Two concepts of causation. In: J. Collins, N. Hall, and L.A. Paul (Eds.), Causation and Counterfactuals. Cambridge, Massachusetts: The MIT Press, 225-276 (2004).

2. C. R. Hitchcock, Of Humean bondage. British Journal for the Philosophy of Science 54, 1-25 (2003).

3. D. Hume, An Enquiry Concerning Human Understanding (Originally published in 1748). Harvard Classics Volume 37, Online Edition (http://eserver.org/18th/hume-enquiry.html). New York: P.F. Collier \& Son (1910).

4. S. Psillos, Causation 83 Explanation. Cambridge: Cambridge University Press (2002).

5. J. Williamson, Causality. In: D. Gabbay and F. Guenthner (Eds.), Handbook of Philosophical Logic, vol. 14. New York: Springer, 1-30 (Forthcoming).

6. J. Williamson, Causal pluralism versus epistemic causality. To appear in Philosophica.

7. A. Chakravartty, Causal realism: Events and processes. Erkenntnis 63, 7-31, (2005).

8. H. Price, Causation in the special sciences: the case for pragmatism. In: D. Constantini, M.C. Galavotti and P. Suppes (Eds.), Stochastic Causality. Stanford: CSLI Publication, 103-120 (2001).

9. H. Price, Causal perspectivalism. In: H. Price and R. Corry (Eds.), Causation, Physics and the constitution of Reality: Russell's republic revisited. Oxford: Clarendon Press, 250-292 (2007). 
10. P. Dowe, Physical Causation. Cambridge, UK: Cambridge University Press (2000).

11. P. Dowe, Causality and conserved quantities: a reply to Salmon. Philosophy of Science 62, 321-333 (1995).

12. J. Kim, Epiphenomenal and supervenient causation. Midwest Studies in Philosophy 9, 257-270 (1984).

13. P. Menzies, Against causal reductionism. Mind 98, 551-574 (1988).

14. P. Dowe, Wesley Salmon's process theory of causality and the conserved quantity theory. Philosophy of Science, 59, 195-216 (1992).

15. S. Webster, Thinking about Biology. Cambridge: Cambridge university press (2003).

16. N. Cartwright, The Dappled World. A Study of the Boundaries of Sciences. Cambridge: University Press (1999).

17. M. Silberstein, Reduction, emergence and explanation. In: P. Machamer and M. Silberstein (Eds.), The Blackwell Guide to the Philosophy of Science, Blackwell Philosophy Guides. Malden, Mass.: Blackwell Publishers, 80-107 (2002).

18. J. L. Mackie, The Cement of the Universe. A study of causation. Oxford: Clarendon Press (1974).

19. P. Dowe, Causes are physically connected to their effects: why preventers and omissions are not causes. In: C. Hitchcock (Ed.), Contemporary Debates in Philosophy of Science. Oxford: Blackwell, 189-196 (2004). 